\title{
The pattern and magnitude of Dietary Free Sugar (DFS) consumption and dental caries in school children: A systematic review
}

A Pakdaman ( $\sim$ pakdaman@sina.tums.ac.ir)

Tehran University of Medical Sciences https://orcid.org/0000-0003-2964-059X

Mahboobi Zeinab

Tehran University of Medical Sciences

Yazdani Reza

Tehran University of Medical Sciences

Azadbakht Leila

Tehran University of Medical Sciences

Research article

Keywords: Diet, Sugar, Dental caries, Children

Posted Date: February 21st, 2020

DOI: https://doi.org/10.21203/rs.2.24169/v1

License: (a) (i) This work is licensed under a Creative Commons Attribution 4.0 International License.

Read Full License 


\section{Abstract}

Background Dietary Free Sugar (DFS) is considered as one of the main risk factor for dental caries development. This systematic review was designed to assess the relationship between pattern and magnitude of Dietary Free Sugar (DFS) consumption and dental caries in school children aged among 6 to 12 years old.

Methods An electronic search of the databases consists of Medline/PubMed, Embase, ISI Web of Science and Scopus was conducted using relevant keywords. Longitudinal studies reporting the relationship between DFS and dental caries focused on school children that were published between 2004 and 2019 in English were selected. The title and abstract of the papers were reviewed by 2 independent researchers and irrelevant papers were excluded. After duplicate removal, the full text of the papers was reviewed. The reference search of the selected papers was performed and finally the quality of included papers assessed using the STROBE check list and the main findings were extracted.

Results From 2120 articles searched, 12 longitudinal studies included in this review. Three articles report insignificant relationship between DFS consumption of and dental caries. However, Dietary Free Sugar and dental caries were significantly associated in the primary and permanent dentition of children. Frequent intake of sweet foods and drinks especially before bed, consumption of processed starches and soft drink were considered as important risk factors while frequent consumption of water and dairy product had protective effect.

Conclusion This study confirms the limitation of dietary free sugar in school children especially in the form of processed starch and sweet snacks especially before bedtime. Still, there is a need for welldesigned studies in this field.

\section{Background}

Diet considered as one of the main risk factors of tooth decay in addition to cariogenic microorganisms, inadequate fluoride exposure, and saliva quantity and quality. Risk indicators such as oral health related knowledge, attitude and behaviour as well as education level, and socio-economic status also have the potential impact on dental caries. Sugar particularly sucrose considered the most important dietary etiological factor for tooth decay [1]. Dietary free sugars (DFS) refers to "all monosaccharides and disaccharides added to foods and sugars naturally present in honey, syrup, fruit juices and concentrates" which increase the risk of dental caries according to the evidence [2].

In 2001, the strong evidence which once existed between sucrose intake and development of dental caries, became weaker in the fluoride era [3]. However, referring to the recent reviews the relationship between sugars and dental caries has been more emphasised both in the field of "pattern" and also "amount" [4]. Accordingly, the reduction of the free sugar intake to less than $10 \%$ of the total energy for better health condition was recommended. This was further advised to reduce the free sugar intake to less than $5 \%$ of total energy intake to prevent dental caries [5]. 
This was along with the WHO recommendations considering by policy makers as a benchmark of sugar consumption at the population level. A review study in 2014 also showed that there was a quantitative relationship between sugar intake and the progressive development of dental caries. The dose-response relationship between dietary sugar and caries further confirmed according to the local data suggesting very low sugar intake as low as $2-3 \%$ of total energy throughout life. This was recommended to be used at the community level with or without optimum level of fluoride [6].

A review study showed that children in the past few decades tend to have energy-dense, nutrient-poor snacks. This is of particular importance because frequent consumption of food with high added and total sugar can lead to high prevalence of overweight and obesity as well as, diabetes and dental caries [7]. Moreover, there is recent evidence on the association of frequent drinking of sugar-sweetened beverages (SSB) and dental caries. This is relating to increase of acidogenic oral bacteria in presence of high-sugar diet [8].

Although many studies reported on the determinants of dental caries, only a few has reported the impact of diet on dental caries specifically in the longitudinal studies and trials on school children [9]. Thus, the purpose of the present systematic review was to assess the studies on the relationship between pattern and magnitude of Dietary Free Sugar (DFS) consumption and dental caries in school children aged between 6 to 12 years old.

\section{Methods}

In this systematic review the Preferred Reporting Items for Systematic Reviews (PRISMA Statement) checklist has been followed [10]. The review was registered in PROSPERO website; with code no.167627. The PICO question of our review was defined as; Population (P): healthy 6-12 years old school children, Intervention (I): exposure to sugary foods or drink, Control (C): low-exposure to sugary items and, Outcome (0): dental caries development as incidence or progression.

\section{Search strategy}

An electronic search of databases for assessing the relationship between "diet" and "dental caries" in "school children" was performed. The following databases were searched including; Embase, PubMed, Scopus and Web of Science for articles published between January 1, 2004 until September 22, 2019. Studies reported the relationship between diet and dental caries were included. In order to manage the search and remove duplicated papers, Endnote Reference Manager Software version X8 was used and duplicated references were removed. PRISMA (Preferred Reporting Items for Systematic Reviews and Meta-Analyses) algorithm [10] was used as the platform for the study. Search strategy for each database presented in Table 1.

\section{Inclusion and Exclusion criteria}


Studies were included with longitudinal or controlled trial design in English language. The search was limited to children aged 6-12. Since the Web of Science database had no filter for age, the key word of "child*" was added. Studies on children with the systemic disease or disorders were excluded. Research articles, letter and conference abstract were included. Unpublished and grey literatures as well as editorials, reviews and other irrelevant records were excluded.

\section{Study Selection and Data Extraction}

Titles and abstracts of the papers obtained from the search were reviewed by two authors (ZM \& AP) independently and unrelated papers were excluded according to the inclusion and exclusion criteria. In case of disagreement, we discussed until reached a consensus. Reference search of the full text articles included were conducted and relevant papers were included.

\section{Quality assessment}

The STROBE (STrengthening The Reporting of OBservational studies in Epidemiology) check list was used for grading of the studies. Two independent reviewers (LA, AP) rated studies according to the checklist. The quality of the selected studies scored according to the STROBE checklist. This check-list contains 22 domains [11]. We scored 1 in each domain if the study met the explanation criteria. The maximum score of 23 was considered as total in our review.

\section{Results}

This review reports the evidence of the studies included on the association between caries incidence/progression using different indices in children aged between 6 - to 12 years old and consumption of Dietary Free Sugar (DFS) as cariogenic diet. From the electronic search, 2120 articled were found and 574 duplicate papers were excluded. After removal of duplicate articles, 1546 papers remained for title review. Title and abstract review was done and 47 papers remained. Finally, after full text review of remained articles, 11 articles were eligible for inclusion in the study. one articles were added after reference search of selected papers. The results of literature search presented in the PRISMA diagram (Figure 1).

In our review 12 studies were included reporting the longitudinal evidence on the association between free sugar consumption and dental caries. Eight studies reported incidence/progression of caries in children under $\mathbf{6}$ years old. Four studies reported the relevant criteria in children over $\mathbf{6}$ years old. In order to record caries experience 4 papers used cavitated vs. non-cavitated status, 1 paper used DMFS index, 2 paper used DMFT/dmft index and 1 paper used ICDAS index. In order to assess diet 2 papers used diet questionnaire, 1 paper used 24-hour recall questionnaire, 1 paper used 3-day diet diary, 1 paper used 4day food record, 1 paper used Food Frequency Questionnaire (FFQ) and 1 paper not reported. The included papers assessed and scored according to STROBE grading system which was presented in Table 2. 


\section{A. Association between free sugar consumption and dental caries in children under 6 years of age}

In the recent study of Ghazal et al. in non-fluoridated American community in 2019, there was no relationship between daily frequency of candy and/or gum, milk, 100\% juice, and added-sugar beverage consumption with new carious lesion. These lesions were measured according to DMFS index in the cohort study of children 6-12 years of age. They found protective effect of water drinking in dental caries development. The study showed previous caries was associated with greater caries hazard after adjusting for the consumption of milk, sugary beverage, and pure (100\%) juice [12].

There was a historical cohort study indicated a significant relationship between dental caries as measured with various indices and diet. In the 5-year historical cohort study by Llena et al. in 2018 assessing 10-year-old children showed that cariogenic diet, especially soft drinks consumption was associated with high DMFT considering cavitated and non-cavitated lesions. In the multivariate analysis, a cariogenic diet, especially soft drinks, was associated with high DMFT and DMFT of the first molar teeth when both cavitated and non-cavitated caries were considered [13].

In the longitudinal study of Hooley et al. in 2012 data derived from the Longitudinal Study of the Australian Children (LSAC), dental problem reported by caregivers was significantly associated with consumption of fatty foods and sweet drinks [14]. In the lowa Fluoride Study (IFS) in 2011 on high socioeconomic Caucasian children who had dental examination in 5 and 8 years old, $37 \%$ had new caries. Having new caries were significantly associated with non-cavitated caries experience at 5 years old; cavitated caries experience at 5 years old; having processed starch at snack frequently; being older and less frequent tooth-brushing [15].

The longitudinal study of Lim et al., in 2008 on a cohort of 369 low SES African-American children aged 3-5 years followed for two years in Detroit demonstrated the relationship between intake of soft drink as risk factor for developing new caries compared with $100 \%$ fruit juice and milk. Children with high consumption of soft drinks had greater chance of having new caries, missing and filled tooth surfaces compared with others. They showed that children who changed the pattern of soft drink consumption from low at baseline to high after 2 years had higher incidence of dental caries [16].

In 7-year longitudinal Finnish study of Ollila et al in 2007, consumption of candies more than once a week and oral hygiene behaviour defined as daily tooth-brushing at age 2 was significantly associated with caries onset in both primary and permanent molars. It was also reported that giving a nursing bottle at night or a pacifier at age 2 years was significantly associated with the risk of caries [9].

The study of Karjalainen et al., in 2015 as part of the STRIP intervention study on diet showed that the risk of caries in adolescents was associated with the high number of sugar intake and Mutans Streptococcus count in childhood at the age of 3 [17]. In the study of Ruttinen et al., in 2004 derived from a prospective, randomized long-term coronary heart disease risk factor intervention trial was conducted as part of STRIP study in Finland to reduce the intake of saturated fat and cholesterol in non-fluoridated city of Turku. Children were followed since 3 years of age. At the mean age of 10 years old dental 
examination was conducted. Caries measured as DMFT and dmft+DMFT scores. In children with higher sucrose intake caries was significantly higher than low sucrose intake group [18].

\section{B. Association between free sugar consumption and dental caries in children over 6 years of age}

Four studies reporting the association between DFS and dental caries in children over 6 years of age. DMFT/dmft index and DMFS index were reported in papers which among them 2 papers were further measured approximal lesions. (Table 2 cont.)

In the study of Levine in 2007, 608 children aged 7 to 11 followed for evaluating dental caries for 4 years. The study subjects filled out a 3-day self-reported diary record at baseline and follow up. Significant relationship was found between current sugar-sweetened beverages consumption and caries. There was significant relationship between bed-time consumption of NMES (Non-Milk Extrinsic Sugars) drinks at the age of 7-11 and development of caries at the age of 11-15 years [19].

The longitudinal study of Kallestal et al, in 2007 on Swedish adolescents with 4 years follow up period showed that low socio-economic status, immigrants from outside Western Europe, those with high candy consumption and having poor oral hygiene had higher caries increment experienced enamel and dentine lesions [20].

There were two studies in this age group which couldn't show any association between DFS and dental caries. In Bruno-Ambrosius study done in 2005, no significant association was found between snacking habit, soft drink and sweet consumption of Swedish girls [21]. Leroy in a 6-year follow up of 7 years old children showed that there was no association between dental caries and consumption of sweets and biscuits in school, consumption of sweet snacks and consumption of sugar-containing drinks [22]. The quality of evidence quality scored according to STROBE check list. 8 studies were rated as good, and 4 as poor. The studies time span in presented in Figure 2.

\section{Discussion}

This systematic review assessed the pattern and magnitude of Dietary Free Sugar (DFS) consumption and dental caries in schoolchildren aged 6 to 12 years. Most studies included in our review showed a significant relationship between sugar consumption in the form of sweet or drink and dental caries using different indices in school children though few studies showed not any association. Consumption of sugary beverage and snack especially when started from early ages were significantly associated with the higher incidence or progression of enamel and dentinal lesions. Having bedtime intake of NMEs drink and food, fatty food, processed starch consumption and early exposure to Streptococcus Mutans in addition to omitting breakfast, lunch or dinner were associated with higher caries incidence or progression. Frequent consumption of water and dairy products were considered as protective factor of caries development. 
The odds ratio was reported in some longitudinal studies. One study reported high association between consumption of processed starch in snack times and caries in children under 6 years of age; odds ratio of 3.87 was reported in the longitudinal study of Chankanka et al., on 5 years old children followed for 4 years [15]. The effect of starch on dental caries has been further emphasized by Halvorsrud et al., in his recent review [23].

In our review the recent study of Ghazal et al., in 2019 following up of American 6-years old children for 6 years reported no significant association between sugar consumption and caries measured on tooth surfaces as DMFS. This study reported the protective effect of water consumption on dental caries [12]. Also the study of Leroy in Belgium in 2005 and Bruno-Ambrosius in Sweden in 2005 reported no significant association between sugar consumption and dental caries. Other papers included in our reviews showed some association between sweet diet and caries measured with different indices in children aged 6-12 years old. In one study only girls were followed and this limits the generalizabiliy of the study [21].

In our review there was strong evidence confirmed the impact of soft drinks on caries development as reported in the study of Hooley et al in 2012 [14]. Despite the fact that some studies did not report any association between consumption of SSB including soft drink and other sugary drinks and caries in the primary and permanent dentition such as the study of Lueangpiansamut, et al., in 2012 [24].

In our review, a significant relationship between sweet food and snacks consumption and dental caries has been reported in most studies. However, 3 studies couldn't show any such association [12, 21, 22]. A 4-years cohort study of Chankanka in 2011, indicated that existing of new cavitated lesions was associated with consumption of processed starches at snack time. This is in line with the study of Hujoel and Lingström in their narrative review in 2017, discussed that fermentable carbohydrates especially sugars are responsible for caries development. The susceptibility of teeth for dental caries in presence of fermentable carbohydrates including sucrose, glucose, fructose, lactose, maltose and starch has been discussed in the literature [25].

Studies included in our review are heterogeneous in terms of age and the follow up period. Studies included in our review reported 2 to 7 years of follow up in studies on children less than 6 years of age. Few studies reported longer period of follow up as part of a randomized controlled trial on other health issues like cholesterol. Moreover, studies included are heterogeneous in terms of indices used to measure caries, which makes the comparison different.

Bedtime consumption of sugary food or drinks reported as a risk factor of caries in different types of studies. There is evidence that sugar consumed before bed increases the risk of caries due to the reduced saliva flow and sustained low plaque pH. The study of Baghlaf et al., in 2017 has been addressing the relationship between dental caries experience and the consumption of foods and drinks containing free sugars at bedtime in 3 to 16 years old children. Despite the low quality of evidence, they found a positive association between dental caries and free sugar consumption at bedtime in children. This systematic review emphasized on more consistent evidence for preschool children compared with older groups [26]. 
Tagi et al., in 2018 showed that children who consumed cariogenic food or drinks between main meals $(p=0.01)$ and within two hours before bedtime $(p=0.04)$ had significantly higher mean caries as measured according to the D4-6MFT index compared with children who did not have this habit [27].

In our review only dietary factors reported however, there are other predisposing factors evaluated in different studies. In the study by Ollila et al, in 2007 eating candies more than once a week and insufficient oral hygiene at two years old were key risk factor for dental caries development in deciduous and permanent molar teeth. They showed that children who used nursing bottle at night for a long time similarly had harmful habits of using candies more than once a week, did not brush their teeth often, and did not use fluoride pills. It can be concluded that most of life style habits of children such as tooth brushing or dietary habits form from first childhood years considered important which was shown in the ten-year cohort study done by Mattila et al in 2005 [28]. Our review confirms the necessity of diet advice along with other preventive measure to control caries in school children which is in line with other preventive measures as discussed in the review study of Pakdaman et al., on caries progression [29].

In our study, a meta-analysis to address the review question was not feasible as the heterogeneous nature of the studies. In our review, there was variability in the caries outcome measure (i.e., DMFS, dmfs, DMFT, dmft, caries, and ICDAS/BASCD criteria). In addition, various dietary outcome measures and tools being used (24-hour recall, FFQ, 4-day food record, 3-day diary, and questionnaire). In addition, there was variation in the follow-up periods.

\section{Strength}

One of the strength of this study is to select high level of evidence including the longitudinal studies. Those studies included as trials of other health factors such as cholesterol also considered. Although association between diet and dental caries was observed in several cross-sectional studies, no study reported just the evidence of cohort studies. Moreover, we used STROBE checklist for grading of the studies. Two independent reviewers scored the included papers according to the checklist. In some studies further analysis of data including survival analysis performed such as the study of Ollila, Ghazal, and Leroy $[9,12,22]$.

\section{Limitations}

Evidence reporting the association between diet and dental caries in schoolchildren are heterogeneous in terms of using different indices to measure caries and diet assessment tools. Moreover, in our systematic review articles published in non-English language, also unpublished and grey literatures have not been included which is considered as limitation. It is important to consider that there are no pure interventional studies on high-sugar diet due to ethical issues despite the fact that in some studies the sample was derived from a trial on other health issues.

\section{Conclusion}


Our review confirms the evidence in the longitudinal studies on the association between Dietary Free Sugar (DFS) consumption and dental caries in schoolchildren. Those children having sugary beverage and snack especially when started from early ages, having bed-time intake of DFS drink or food, processed starch consumption were associated with higher caries incidence. Longitudinal studies confirmed the potential risk of developing caries in children with high sugar consumption, still, there is a need for well-designed studies in this field.

\section{Abbreviations}

DFS: Dietary Free Sugar; STROBE: Strengthening The Reporting of OBservational studies in Epidemiology; WHO: World Health Organization; PRISMA: Preferred Reporting Items for Systematic Reviews and MetaAnalyses; PICO: Population, Intervention, Control or Comparison, Outcome; DMFT/S: Decayed, Missed, Filled Tooth/Surface in permanent dentition; DeMFS: Decayed (enamel), Missed, Filled Surface in permanent dentition; dmft: decayed, missed, filled tooth in primary dentition; ICDAS: International Caries Detection and Assessment System; FFQ: Food Frequency Questionnaire; LSAC: Longitudinal Study of the Australian Children; IFS: lowa Fluoride Study; SES: Socio-Economic Status; STRIP: Special Turku Coronary Risk Factor Intervention Project; NMES: Non-Milk Extrinsic Sugars; SSB: Sugar sweetened Beverage; BASCD: British Association for the Study of Community Dentistry; HR: Hazard Ratio; PR: Prevalence Ratio; OR: Odds Ratio; IRR: Incidence Rate Ratio.

\section{Declarations}

\section{Ethics approval and consent to participate}

It is not applicable as this paper is a systematic review.

\section{Consent to publish}

Not applicable.

\section{Availability of data and materials}

All articles included in this review containing data supporting the findings are in the reference list.

\section{Competing interests}

The authors have no competing interests.

\section{Funding}

This study is conducted as part of PhD thesis supported by Tehran University of Medical Sciences.

\section{Authors' contributions}


Both authors (ZM and AP) independently assessed the title, abstract and full text of the selected papers included in this systematic review.

ZM: literature search, extracting data, interpreting data, preparing the manuscript.

AP: conceptualization of the study, design of the research, literature search, extracting data, interpreting data, preparing the manuscript.

RY: conceptualization of the study, interpreting data, drafting the manuscript.

LA: conceptualization of the study, interpreting data, drafting the manuscript.

LA and AP independently assessed the full text papers and graded it according to the STROBE checklist.

All authors have read and approved the manuscript.

\section{Acknowledgements}

The authors acknowledge the help of Dr. Akbar Shafiee Specialist in Community Medicine (Department of Cardiovascular Research, Tehran Heart Center, Tehran University of Medical Sciences, Tehran, Iran) in guiding the database search of this study. This study is conducted as part of PhD thesis supported by Tehran University of Medical Sciences.

\section{References}

1. Fejerskov O, Nyvad B, Kidd E. Dental caries: the disease and its clinical management, 3rd edn: John Wiley \& Sons; 2015.

2. Moynihan P, Petersen PE. Diet, nutrition and the prevention of dental diseases. Public Health Nutr. 2004, 7(1A):201-226.

3. Burt BA, Pai S. Sugar consumption and caries risk: a systematic review. J Dent Educ. 2001, 65(10):1017-1023.

4. Moynihan P, Kelly S. Effect on caries of restricting sugars intake: systematic review to inform WHO guidelines. J Dent Res. 2014, 93(1):8-18.

5. Moynihan P. Sugars and Dental Caries: Evidence for Setting a Recommended Threshold for Intake. Adv Nutr. 2016, 7(1):149-156.

6. Sheiham A, James WPT. A reappraisal of the quantitative relationship between sugar intake and dental caries: the need for new criteria for developing goals for sugar intake. BMC Public Health. 2014, 14(1):863.

7. Larson N, Story M. A review of snacking patterns among children and adolescents: what are the implications of snacking for weight status? Child Obes. 2013, 9(2):104-115.

8. Bleich SN, Vercammen KA. The negative impact of sugar-sweetened beverages on children's health: an update of the literature. BMC Obes. 2018, 5(1):6. 
9. Ollila P, Larmas M. A seven-year survival analysis of caries onset in primary second molars and permanent first molars in different caries risk groups determined at age two years. Acta Odontol Scand. 2007, 65(1):29-35.

10. Transparent reporting of systematic reviews and meta-analyses. Available from: http://www.prismastatement.org/. Accesed 2 Feb 2019.

11. White RG, Hakim AJ, Salganik MJ, Spiller MW, Johnston LG, Kerr L,et al. Strengthening the reporting of observational studies in epidemiology for respondent-driven sampling studies: "STROBE-RDS" statement. J Clin Epidemiol. 2015, 68(12):1463-1471.

12. Ghazal TS, Levy SM, Childers NK, Carter KD, Caplan DJ, Warren JJ,et al. Survival analysis of caries incidence in African-American school-aged children. J Public Health Dent. 2019, 79(1):10-17.

13. Llena C, Calabuig E. Risk factors associated with new caries lesions in permanent first molars in children: a 5-year historical cohort follow-up study. Clin Oral Investig. 2018, 22(3):1579-1586.

14. Hooley M, Skouteris $H$, Millar L. The relationship between childhood weight, dental caries and eating practices in children aged 4-8 years in Australia, 2004-2008. Pediatr Obes. 2012, 7(6):461-470.

15. Chankanka O, Marshall TA, Levy SM, Cavanaugh JE, Warren JJ, Broffitt B, et al. Mixed dentition cavitated caries incidence and dietary intake frequencies. Pediatr Dent. 2011, 33(3):233-240.

16. Lim S, Sohn W, Burt BA, Sandretto AM, Kolker JL, Marshall TA, et al. Cariogenicity of soft drinks, milk and fruit juice in low-income african-american children: a longitudinal study. J Am Dent Assoc. 2008, 139(7):959-967;

17. Karjalainen S, Tolvanen M, Pienihäkkinen K, Söderling E, Lagström H, Simell O, et al. High sucrose intake at 3 years of age is associated with increased salivary counts of mutans streptococci and lactobacilli, and with increased caries rate from 3 to 16 years of age. Caries Res. 2015, 49(2):125132.

18. Ruottinen S, Karjalainen S, Pienihäkkinen K, Lagström H, Niinikoski H, Salminen M, et al. Sucrose intake since infancy and dental health in 10-year-old children. Caries Res. 2004, 38(2):142-148.

19. Levine RS, Nugent ZJ, Rudolf MC, Sahota P. Dietary patterns, toothbrushing habits and caries experience of schoolchildren in West Yorkshire, England. Community Dent Health. 2007, 24(2):82-87.

20. Källestål C, Fjelddahl A. A four-year cohort study of caries and its risk factors in adolescents with high and low risk at baseline. Swed Dent J. 2007, 31(1):11-25.

21. Bruno-Ambrosius K, Swanholm G, Twetman S. Eating habits, smoking and toothbrushing in relation to dental caries: a 3-year study in Swedish female teenagers. Int J Paediatr Dent. 2005, 15(3):190196.

22. Leroy R, Bogaerts K, Lesaffre E, Declerck D. Multivariate survival analysis for the identification of factors associated with cavity formation in permanent first molars. Eur J Oral Sci. 2005, 113(2):145152.

23. Halvorsrud K, Lewney J, Craig D, Moynihan P. Effects of starch on oral health: systematic review to inform WHO Guideline. J Dent Res. 2019, 98(1):46-53. 
24. Lueangpiansamut J, Chatrchaiwiwatana S, Muktabhant B, Inthalohit W. Relationship between dental caries status, nutritional status, snack foods, and sugar-sweetened beverages consumption among primary schoolchildren grade 4-6 in Nongbua Khamsaen school, Na Klang district, Nongbua Lampoo Province, Thailand. J Med Assoc Thai. 2012, 95(8):1090-1097.

25. Hujoel PP, Lingström P. Nutrition, dental caries and periodontal disease: a narrative review. J Clin Periodontol. 2017, 44:S79-S84.

26. Baghlaf K, Muirhead V, Moynihan P, Weston-Price S, Pine C. Free sugars consumption around bedtime and dental caries in children: a systematic review. JDR Clin Trans Res. 2018, 3(2):118-129.

27. Taqi M, Razak IA, Ab-Murat N. Sugar consumption and caries occurrence among Pakistani school children. J Pak Med Assoc. 2018, 68(10):1483-1487.

28. Mattila M-L, Rautava P, Aromaa M, Ojanlatva A, Paunio P, Hyssälä L,et al. Behavioural and demographic factors during early childhood and poor dental health at 10 years of age. Caries Res. 2005, 39(2):85-91.

29. Pakdaman A, Montazeri A, Evans R. Deciduous dentition approximal caries lesion progression and regression following preventive treatment: literature review. Aust Dent J. 2018, 63(4):422-428.

\section{Tables}


Table 1.

Embase 361 ('diet'/exp OR 'cariogenic diet'/exp OR 'carbohydrate diet'/exp OR 'carbohydrate loading diet'/exp OR 'free sugar*':ti,ab,kw OR 'added sugar*':ti,ab,kw OR 'sucrose'/exp OR 'sugar'/exp OR 'drinking behavior'/exp OR 'sugar intake'/exp OR 'eating habit'/exp) AND ('dental health'/exp OR ' $\mathrm{dmf}^{1}{ }^{1}$ index'/exp OR 'dental caries'/exp) AND (2004:py OR 2005:py OR 2006:py OR 2007:py OR 2008:py OR 2009:py OR 2010:py OR 2011:py OR 2012:py OR 2013:py OR 2014:py OR 2015:py OR 2016:py OR 2017:py OR 2018:py OR 2019:py) AND ('article'/it OR 'article in press'/it OR 'conference abstract'/it OR 'letter'/it) AND ([preschool]/lim OR [school]/lim) AND [English]/lim

PubMed 357 Search (((((((("Diet"[Mesh]) OR "Diet, Cariogenic"[Mesh]) OR "Diet, Carbohydrate Loading"[Mesh])) OR (("Dietary Sugars"[Mesh]) OR "Dietary Sucrose"[Mesh])) OR (("added sugar"[Title/Abstract]) OR "free sugar"[Title/Abstract])) OR drinking behavior[Title/Abstract]) OR eating habit[Title/Abstract])) AND (((((("Dental Caries"[Mesh]) OR "Tooth Demineralization"[Mesh])) OR "DMF Index"[Mesh]) OR "Oral Health"[Mesh]) OR Caries[Title/Abstract]) OR "Tooth decay"[Title/Abstract]) Filters: Congress; Journal Article; Letter; Publication date from 2004/01/01; English; Child: 6-12 years

Scopus 269 ((TITLE-ABS-KEY(diet) OR TITLE-ABS-KEY("cariogenic diet") OR TITLE-ABSKEY("carbohydrate diet") OR TITLE-ABS-KEY("sugar*") OR TITLE-ABSKEY(sucrose) OR TITLE-ABS-KEY("eating habit") OR TITLE-ABS-KEY("drinking behavior") OR TITLE-ABS-KEY("added sugar") OR TITLE-ABS-KEY("free sugar"))) AND ((TITLE-ABS-KEY("dental caries") OR TITLE-ABS-KEY("tooth demineralization") OR TITLE-ABS-KEY("tooth decay") OR TITLE-ABS-KEY("DMF index") OR TITLE-ABS-KEY (caries) OR TITLE-ABS-KEY("oral health") OR TITLEABS-KEY("dental health"))) AND (LIMIT-TO (PUBYEAR,2019) OR LIMIT-TO (PUBYEAR,2018) OR LIMIT-TO (PUBYEAR,2017) OR LIMIT-TO (PUBYEAR,2016) OR LIMIT-TO (PUBYEAR,2015) OR LIMIT-TO (PUBYEAR,2014) OR LIMIT-TO (PUBYEAR,2013) OR LIMIT-TO (PUBYEAR,2012) OR LIMIT-TO (PUBYEAR,2011) OR LIMIT-TO (PUBYEAR,2010) OR LIMIT-TO (PUBYEAR,2009) OR LIMIT-TO (PUBYEAR,2008) OR LIMIT-TO (PUBYEAR,2007) OR LIMIT-TO (PUBYEAR,2006) OR LIMIT-TO (PUBYEAR,2005) OR LIMIT-TO (PUBYEAR,2004)) AND ( LIMIT-TO ( DOCTYPE,"ar" ) OR LIMIT-TO (DOCTYPE,"cp") OR LIMIT-TO ( DOCTYPE,"le" ) ) AND (LIMIT-TO (EXACTKEYWORD,"Children" ) OR LIMIT-TO (EXACTKEYWORD,"School Child" ) ) AND ( LIMIT-TO (LANGUAGE,"English" ) )

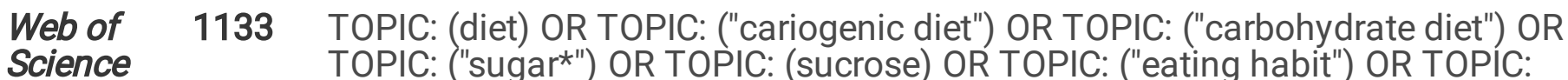
("drinking behavior") OR TOPIC: ("free sugar") OR TOPIC: ("added sugar") Indexes=SCI-EXPANDED, SSCl, A\&HCl, CPCl-S, CPCl-SSH, ESCI Timespan=All years AND TOPIC: ("dental caries") OR TOPIC: ("tooth demineralization") OR TOPIC: ("tooth decay") OR TOPIC: (caries) OR TOPIC: ("DMF*") OR TOPIC: ("oral health") OR TOPIC: ("dental health")

${ }^{1}$ In the Embase database the "dmf" index included both "DMF and dmf" index.

Due to technical limitations, table 2 is only available as a download in the supplemental files section.

\section{Figures}



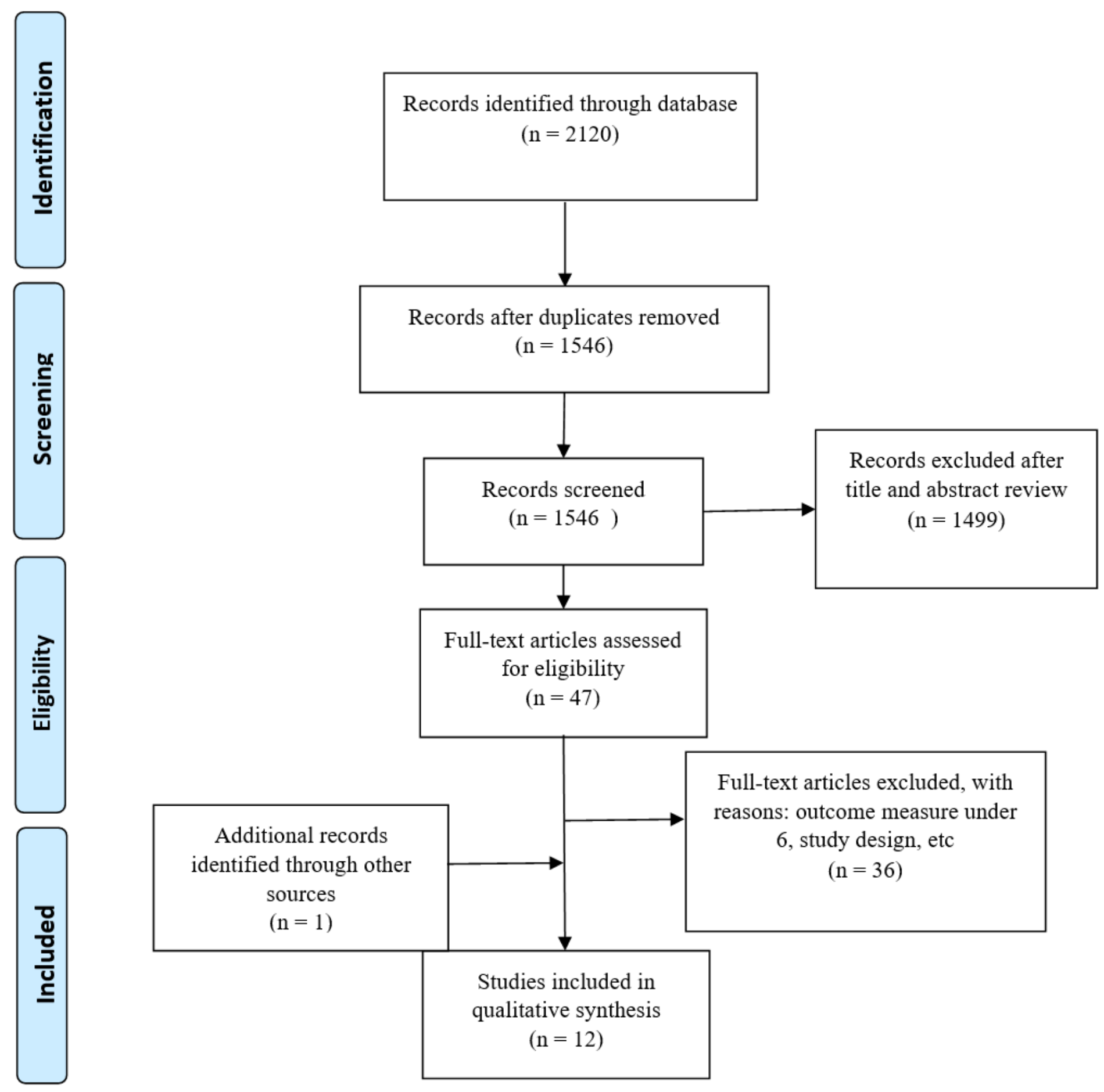

\section{Figure 1}

Flow chart of the study according to PRISMA. 


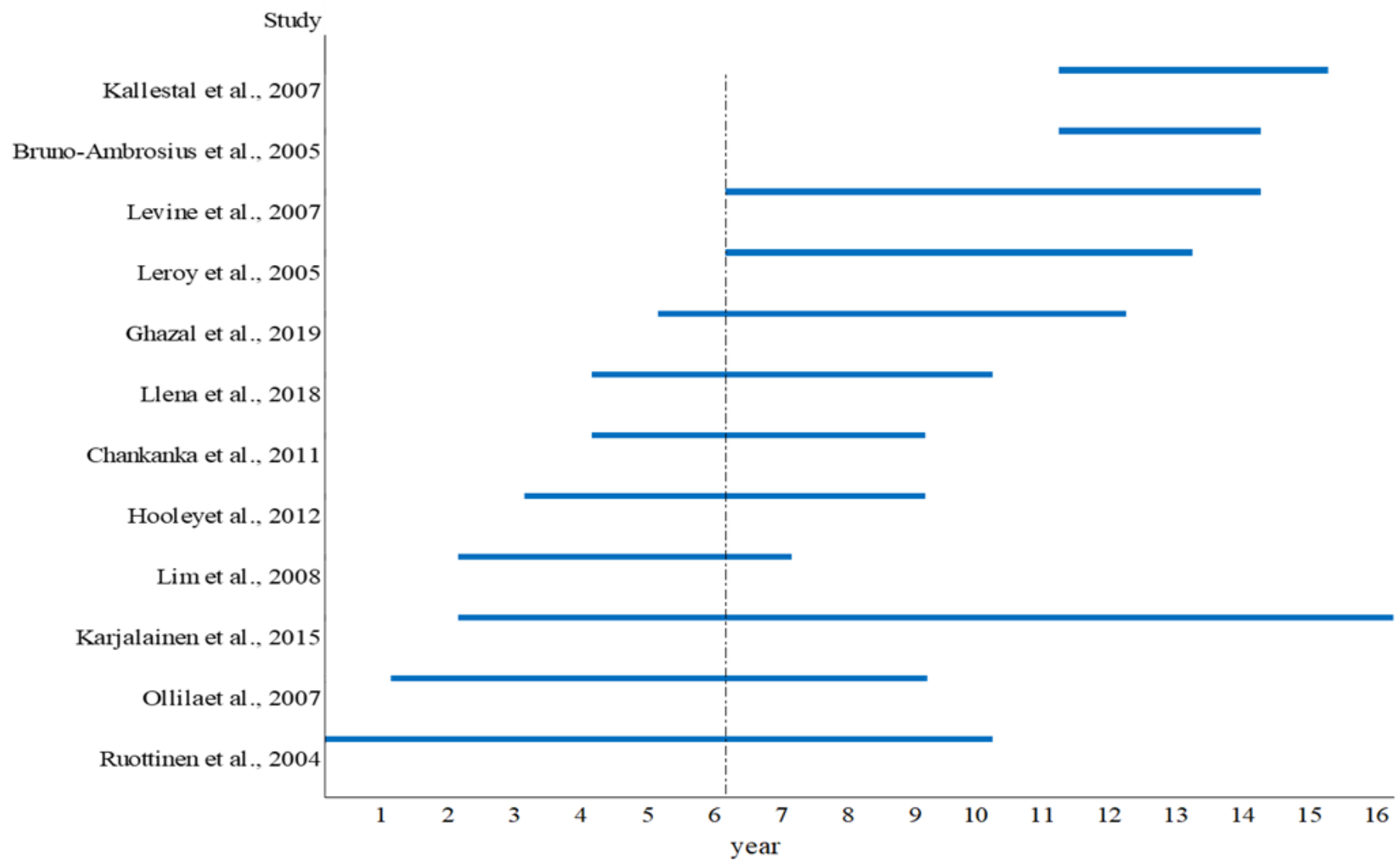

Figure 2

Summary of the study duration included in the systematic review.

\section{Supplementary Files}

This is a list of supplementary files associated with this preprint. Click to download.

- Table2cont.edited2.xls

- Table2edited2.xls 